\title{
Spiral shock detection on eclipse maps: Simulations and observations ${ }^{\star}$
}

\author{
E. T. Harlaftis ${ }^{1}$, R. Baptista ${ }^{2}$, L. Morales-Rueda ${ }^{3}$, T. R. Marsh ${ }^{3}$, and D. Steeghs ${ }^{4}$ \\ ${ }^{1}$ Institute of Space Applications and Remote Sensing, National Observatory of Athens, PO Box 20048, Athens 11810, Greece \\ 2 Departamento de Física, Universidade Federal de Santa Catarina, Campus Trindade, 88040-900 Florianópolis, Brazil \\ e-mail: bap@fsc.ufsc.br \\ 3 Department of Physics and Astronomy, Southampton University, Southampton SO17 1BJ, UK \\ e-mail: lmr@astro.soton.ac.uk, trm@astro.soton.ac.uk \\ ${ }^{4}$ High Energy Astrophysics Division, Center for Astrophysics, MS-67, 60 Garden Street, Cambridge, MA 02138, USA \\ e-mail: dsteeghs@head-cfa.cfa.harvard.edu
}

Received 7 November 2003 / Accepted 27 December 2003

\begin{abstract}
We perform simulations in order to reveal the effect of observational and physical parameters on the reconstruction of a spiral structure in an accretion disk, using eclipse mapping techniques. We show that a model spiral structure is smeared to a "butterfly"-shape structure because of the azimuthal smoothing effect of the technique. We isolate the effects of phase resolution, signal-to-noise ratio and accurate centering of the eclipse at zero phase. We further explore disk emissivity factors such as dilution of the spiral structure by the disk light and relative spiral arm difference. We conclude that the spiral structure can be satisfactorily recovered in accretion disk eclipse maps with phase resolution $|\Delta \phi| \leq 0.01, S / N>25$ and zero phase uncertainty $|\Delta \phi| \leq 0.005$, assuming the two spiral arms have similar brightness and contribute $\geq 30 \%$ to the total disk light. Under the light of the performed simulations, we present eclipse maps of the IP Peg accretion disk reconstructed from eclipse light curves of emission lines and continuum during the outburst of August 1994, where spiral shocks were detected with the aid of Doppler tomography (Morales-Rueda et al. 2000). We discuss how the detection of spirals shocks with eclipse mapping is improved with the use of velocity-resolved eclipse light curves which do not include any contaminating low-velocity emission.
\end{abstract}

Key words. stars: novae, cataclysmic variables - stars: individual: IP Pegasi - accretion, accretion disks - shock waves methods: data analysis - line: formation - techniques: photometric

\section{Introduction: Spiral shocks in cataclysmic variables}

Cataclysmic variables (CVs) are binaries where an accretion disk is formed around the white dwarf from gas escaping a companion red dwarf (Warner 1995). Their study is attractive for understanding binary formation and evolution and the physical processes occuring in the accretion flow. For example, the semi-regular radiation outbursts from the accretion disks around white dwarfs in the CV sub-class of the dwarf novae are thought to be driven by a thermal instability within the disk (Hameury 2003 and references therein). The optical radiation pattern on the accretion disk is asymmetric and is dominated either by the "bright spot" caused by the impact of the gas stream on the accretion disk in quiescence (Marsh 1989) or the "spiral

Send offprint requests to: E. T. Harlaftis, e-mail: ehh@space.noa.gr

* Based on observations made with the Isaac Newton and William Herschel telescopes operated on the island of La Palma by the Isaac Newton Group in the Spanish Observatorio del Roque de los Muchachos of the Instituto de Astrofisica de Canarias. structure" raised by tides from the companion star on the outer accretion disk during outburst (Steeghs 2001; Boffin 2001).

IP Pegasi is an eclipsing dwarf nova and one of the best studied CVs thus providing a paradigm for the accretion disk model. Spiral shocks in its accretion disk have been found throughout the various stages of an outburst (rise, maximum, decline; Steeghs et al. 1997; Harlaftis et al. 1999; and MoralesRueda et al. 2000, respectively), using the Doppler tomography technique (Marsh \& Horne 1988). The phase-resolved spectra in Harlaftis et al. (1999), which were used to reconstruct the Doppler maps - two-dimensional velocity maps - of the spiral shocks, were also used to make eclipse light curves in various emission lines. The light curves (integrated intensity versus phase) were then analysed using the eclipse mapping technique (Baptista 2001) in order to reconstruct the surface brightness distribution of the accretion disk and thus define the spatial extent of the spiral arms (Baptista et al. 2000). Direct comparison of the Doppler (velocity) and eclipse (spatial) maps showed that the outer disk - largely affected by the velocity field of the spiral shocks - in IP Pegasi is largely non-Keplerian and 
that the spiral arms extend between $0.2-0.6 R_{L_{1}}$, contributing between $16-30 \%$ of the total line flux.

Here, we address the parameters affecting the reconstruction of the spiral structure using the eclipse mapping technique. We undertake simulations in order to demonstrate the effect on the detectability of spiral arms in situations with an unfavorable combination of low $S / N$, low time resolution, and the dilution of the shock light by the disk. Hence, we define the observational requirements for future observations of spiral shocks using the eclipse mapping technique. Finally, in the light of the simulations performed we interpret the eclipse maps built from light curves of IP Pegasi during outburst (Morales-Rueda et al. 2000). This is an epoch where the spiral shocks have been excited in the accretion disk, as shown by Doppler tomography.

\section{Simulations}

Doppler tomography of IP Peg has clearly revealed the disk spiral structure on a number of occassions and showed its strength in imaging sub-structures within the accretion disk (Steeghs 2001). However, the disk spiral structure of IP Peg is, apparently, not imaged as clearly using the eclipse mapping technique (Baptista et al. 2000), even though the eclipse light curves are from the same outburst maximum (November 1996). There, the same phase-resolved spectra show one of the best examples of spiral shocks in an accretion disk using Doppler tomography as the reconstruction technique (Harlaftis et al. 1999). As a consequence, we have performed a series of simulations in order to better understand the structures reconstructed with eclipse mapping. The reconstructions were obtained using the PRIDA code (Baptista \& Steiner 1993), which computes an eclipse map as an array of $51 \times 51$ pixels, with a size of $2 R_{L_{1}}$ and centred on the white dwarf. Any additional uneclipsed component can also be modelled as a constant flux. We adopt the binary geometry of Wood \& Crawford (1986) with $(i, q)=\left(81^{\circ}\right.$, 0.5 ), where $i$ is the inclination and $q$ is the mass ratio (see also Sect. 3.1). For the reconstructions we adopted the default of limited azimuthal smearing (Rutten et al. 1992), which is better suited for recovering asymmetric structures than the original default of full azimuthal smearing (e.g., Baptista 2001). For more details on the technique, see Baptista (2001). Using a model map with a symmetric disk and a spiral structure, we test the limits of the technique in resolving the spiral arms. The parameters we change are either defined by the observing technique, geometrical constraints or physical processes:

- spiral structure orientation;

- phase resolution;

- signal-to-noise ratio;

- accurate zero phase of mid-eclipse;

- spiral structure dilution by the symmetric disk component;

- brightness difference between the two spiral arms.

For the simulations, we use as input a model map consisting of two spirals superimposed on a symmetric disk. The spirals have a radial width of $0.08 R_{L_{1}}$ and a radial range between $0.2-$ $0.6 R_{L_{1}}$, as found in Baptista et al. (2000). The trace of the spiral is defined by,

$R_{\text {spiral }}(\theta)=\left(\theta-\theta_{0}\right) / \alpha$

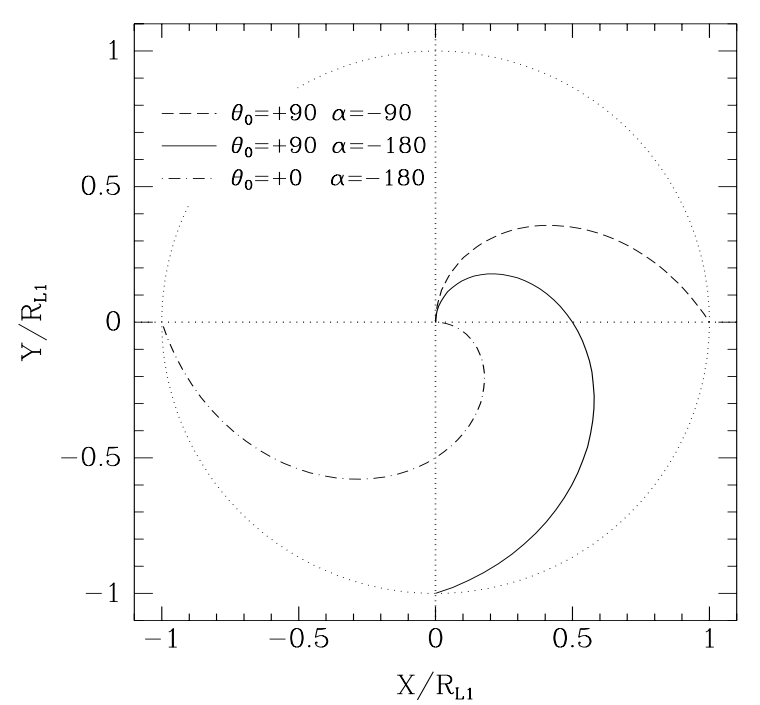

Fig. 1. The spiral arm definition using the parameters $\theta_{0}$, the angle between the tangent to the spiral pattern (extrapolated to the disk centre at $\left.R_{\text {spiral }}=0 R_{L_{1}}\right)$ and the line-of-centres, and $\alpha\left(=-180^{\circ}\right)$, the opening angle of the spiral, or the change in angle $\left(\theta-\theta_{0}\right)$ it takes to go from $R_{\text {spiral }}=0$ to $R_{\text {spiral }}=R_{L_{1}}$. The line-of-centres is the positive direction of the $x$-axis and the angles are measured counter-clockwise.

where $\theta_{0}$ is the angle between the tangent to the spiral pattern (extrapolated to the disk centre at $R_{\text {spiral }}=0 R_{L_{1}}$ ) and the lineof-centres and increases counter-clockwise in steps of $30^{\circ}$, using as a reference the observed rotation angle at $46^{\circ}$ (Baptista et al. 2000); $\theta$ is the angle between the tangent to the spiral pattern at $0<R_{\text {spiral }}<R_{L_{1}}$ and $\alpha\left(=-180^{\circ}\right)$ is the opening angle of the spiral, the change in angle $\left(\theta-\theta_{0}\right)$ from $R_{\text {spiral }}=0$ to $R_{\text {spiral }}=0.6 R_{L_{1}}$. Note that the above definition is for linear spiral arms (see Binney \& Tremaine 1988 for logarithmic spiral arms). Figure 1 shows graphically the spiral definition with $\theta_{0}$ and $\alpha$ as defined above. Setting a small (large) $\alpha$ results in a tightly wound (open) spiral arm. Our model map in Fig. 2 has an underlying symmetric disk containing $40 \%$ of the total flux and a flat brightness distribution which drops sharply at $R=0.6 R_{L_{1}}$ following a $\exp \left[-\left|\left(r-r_{0}\right) / \mathrm{d} r\right|^{3}\right]$ law. The spirals are also cut off with the same radial dependency, both at the inner and outer radius.

\subsection{Spiral structure orientation $\left(\theta_{0}\right)$}

In Fig. 2, we present model maps of spiral arms at different rotation angles which we use as input images for the simulations, so that we compare the predicted eclipse light curves with the observed ones. The dashed lines in the left hand panels show the contribution of the two spiral arms to the light curves; the dash-dotted lines show the contribution of the symmetric disk component to the light curves; and the solid lines show the total light curve. The rotation angle $\theta_{0}$ of the spiral arm main axis is indicated in each panel. The model surface brightness distribution maps are shown in a logarithmic grayscale (dark regions are brighter). There, the dotted lines depict the primary Roche lobe, the gas stream trajectory, and the disk radii at 0.2 and $0.6 R_{L_{1}}$. A cross marks the disk centre. The stars rotate counter-clockwise and the secondary is to the right of each 

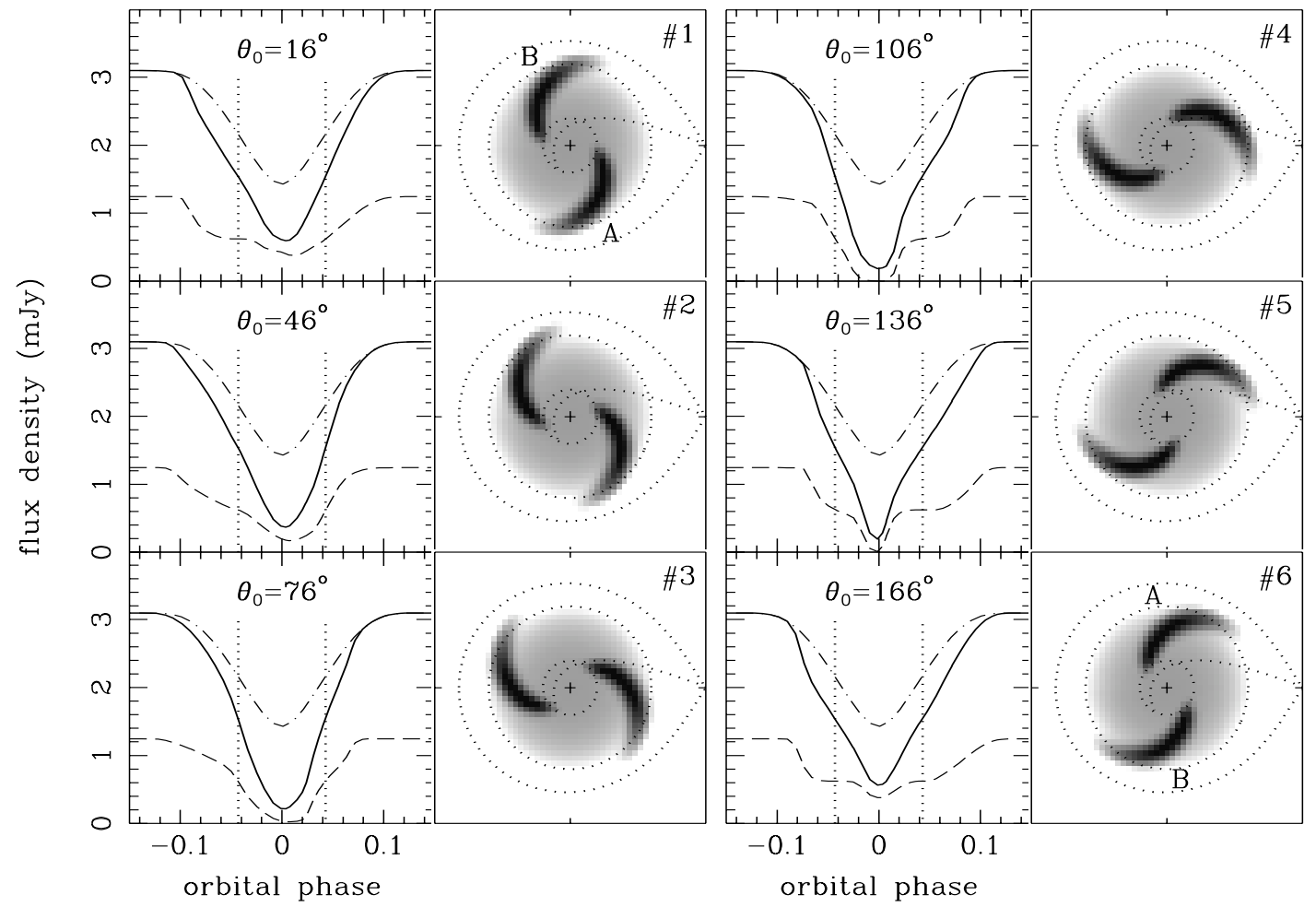

Fig. 2. A model accretion disk with a spiral structure at different rotation angles and the corresponding total, disk, and spiral light curves. The model surface brightness distributions are shown in a logarithmic grayscale (dark regions are brighter). Dotted lines depict the primary Roche lobe, the gas stream trajetory, and the disk radius at 0.2 and $0.6 R_{L_{1}}$ (extent of observed spiral shocks; Baptista et al. 2000). A cross marks the disk centre. The stars rotate counter-clockwise and the secondary is to the right of each map. In the left-hand panels, vertical dotted lines depict the ingress/egress phases of the disk centre (white dwarf). The rotation angle of the spiral arm axis is indicated in each panel (see also text). We adopt the model with an angle $\alpha=46^{\circ}$ which corresponds to the spirals observed (Baptista et al. 2000; Harlaftis et al. 1999). The model with an angle $\theta_{0}=136^{\circ}$ produces an eclipse shape which resembles that observed by Baptista et al. (2002). The two spiral arms of equal brightness are labeled "A" and "B" in two of the panels. The dashed lines in the left-hand panels show the contribution of the two spiral arms to the light curves; the dot-dashed lines show the contribution of the symmetric disk component to the light curves; and the solid lines show the total light curve. For visualization purposes, the curve of the symmetric disc component is vertically shifted to match the out-of-eclipse level of the total light curve. Note that the eclipse shape is indeed affected by the rotation of the spiral structure.

panel. The two spiral arms, of equal brightness, are labeled "A" and "B" in two of the panels. The change in the shape of the eclipse is notable as the spiral arms rotate from $16^{\circ}$ to $166^{\circ}$. The wider eclipse ingress shifts to a wider egress of the spiral arms. The spiral arm light curves show best the change in eclipse shape with rotation angle. Reconstructions obtained from these light curves confirm the results of previous simulations (Baptista et al. 2000), namely, that the orientation and radial position of the arms are well recovered in the eclipse maps. The panels present spiral models for increasing steps of $30^{\circ}$ starting at $16^{\circ}$. The eclipse shape of the spiral model with rotation angle $46^{\circ}$ resembles best the observed eclipse maps presented here and in Baptista et al. (2000). This is clearer in the next figure which presents the simulation results with the phase resolution of the observed data. The eclipse shape of the spiral model with $\theta_{0}=136^{\circ}$ resembles the eclipse light curves of Baptista et al. (2002).

\subsection{Phase resolution $(\Delta \phi)$ and $S / N$ ratio}

Figure 3 shows the effect of reducing the phase resolution of the light curve on the map reconstructions. These and all following simulations were performed with the adopted spiral model $\left(\theta_{0}=46^{\circ}\right)$. The left hand panels show data light curves of $S / N=50$ for three phase resolutions: $\Delta \phi=0.005,0.010$ and 0.015 cycles. Gaussian noise has been added in the light curves. Note that the phase resolution of $\Delta \phi=0.010$ is similar to that of the average light curves extracted from the blue spectra of the August 1994 outburst (see Sect. 3). The right-hand panels show the asymmetric part of the resulting eclipse maps in logarithmic greyscale for $S / N=50$ and 25, respectively. The asymmetric component of each eclipse map is computed as follows. The radial intensity profile is separated into 9 radial bins of equal width. We then sort the intensities in each bin, and fit a smooth spline function to the lower quartile of the intensities in each bin. The fit is then subtracted from each pixel and the resulting map is written as the asymmetric component. We practically remove the baseline of the radial profile, leaving all azimuthal structure in the asymmetric map. We further set to zero all negative intensities in the asymmetric map.

The quality of the reconstruction is sensitive to the phase resolution of the data. The light curve with the highest phase resolution and best $S / N$ shows that the spiral structure is considerably blurred to a "butterfly" pattern, as a consequence of 

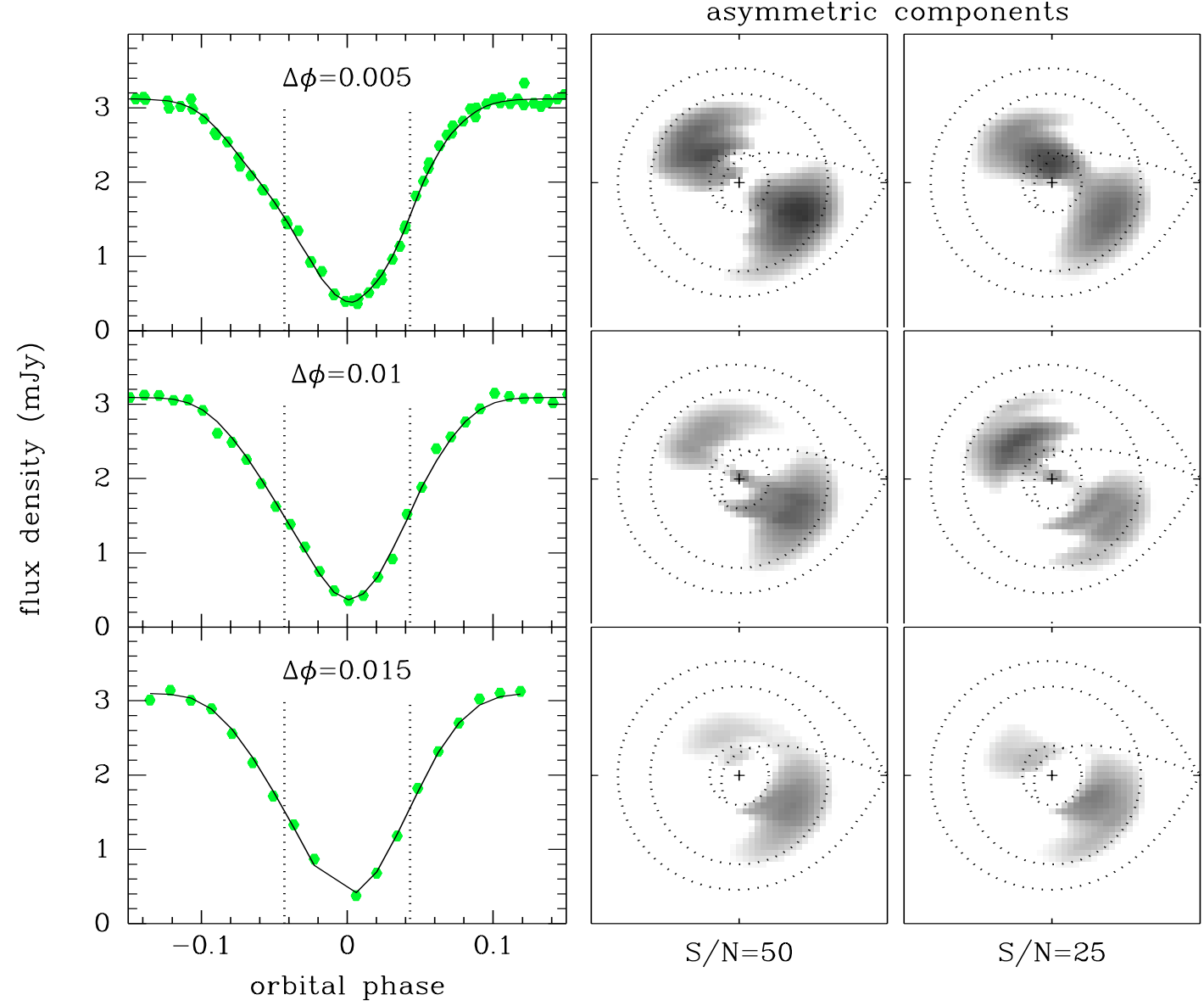

Fig. 3. The effect of the phase resolution of the light curve on the image reconstructions. The left-hand panels show data light-curves from the second panel of Fig. 2 for three phase resolutions: $\Delta \phi=0.005,0.01$ and 0.015 cycles. The corresponding asymmetric parts of the eclipse maps for $S / N=50$ and 25 are presented in logarithmic grayscale.

the azimuthal smearing effect of the eclipse mapping method. Poor phase resolution $(\Delta \phi=0.015)$ results in spiral arms with poorer definition, since the gradient change in the eclipse light curve shape - which testifies the presence of the spirals - is poorly sampled. The noise masks the ingress/egress of the spiral arms and the eclipse mapping method produces a V-shaped fairly symmetric model light curve of constant ingress/egress slope which will drive the solution to a more symmetric configuration by smearing the spiral arms. Thus, the spirals cannot be resolved on the map if the light curves have low $S / N$ (or are affected by significant disk flickering).

\subsection{Accurate zero phase of mid-eclipse $\left(\Delta \phi_{0}\right)$}

In Fig. 4 we apply additional phase offsets to the eclipse light curves in order to explore the effect on the spiral arms of the uncertainty on the exact zero phase ( 0.006 cycles for IP Peg; see Sect. 3). Small errors in the mid-eclipse, $\Delta \phi_{0} \simeq 0.005$ cycles, lead to distortions in the eclipse map by changing the azimuthal position and extension of the spiral arms. Positive (negative) phase offsets displace both arms to the trailing (leading) side of the primary Roche lobe and also reduce the strength of the blue (red) arm, thus severely distorting the disk light distribution. A phase offset of -0.010 to the light curves is sufficient to push the light towards a single segment of the outer disk, in fact where the blue spiral arm lies at. This exercise emphasizes the importance of knowing the value of $\phi_{0}$ with good accuracy.

\subsection{Spiral structure dilution by the symmetric disk component}

Figure 5 presents the test results of the spiral structure light dilution caused by a symmetric disk component. The left-hand panels show model light curves (with phase resolution of $\Delta \phi=$ 0.01 and $S / N=50)$ for disk models $\left(\theta=46^{\circ}\right)$ where the spiral structure contributes $40,30,20$ and 10 per cent of the total flux. The right-hand panels show the asymmetric components - after subtracting the symmetric map from the total intensity map - of the corresponding eclipse maps for $S / N=50$ and 25 , respectively. At small fractional contributions (10\%), the spiral structure is significantly diluted by the superimposed symmetric disk light and the "butterfly"-like pattern (bottom panels) is smeared to a "crescent"-like pattern at the outer disk facing the $L_{1}$ point. Provided that the spiral structure contributes a significant fraction to the total light $\left(f_{\text {spiral }} / f_{\text {total }}>0.2\right)$, the two-arm structure is then reasonably recovered as a "butterfly" pattern.

Estimating the contribution of the spirals from the ratio of the flux in the asymmetric map component and that of the total 


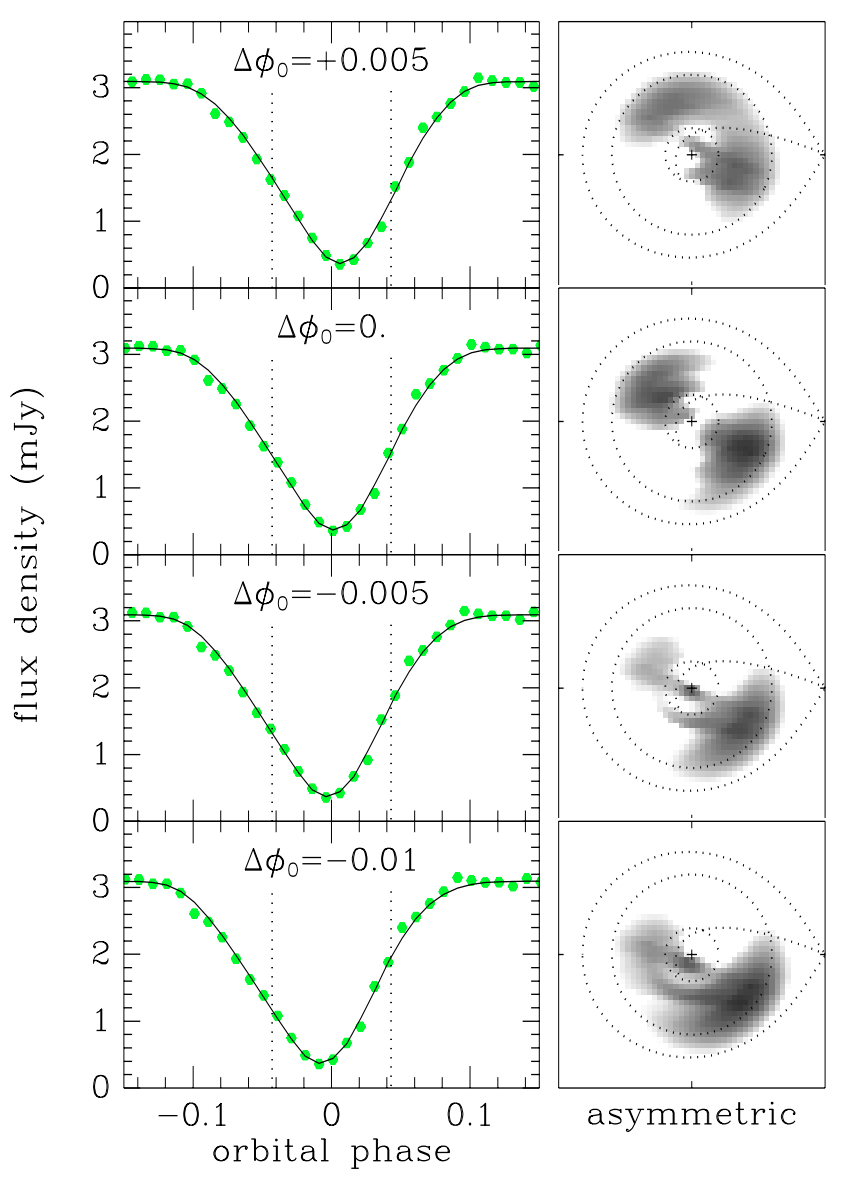

Fig. 4. The effects of accurate phasing of the mid-eclipse (phase offset $\Delta \phi_{0}$ ) on the reconstructions. The value of $\Delta \phi_{0}$ is indicated in each panel; the asymmetric component of the corresponding eclipse maps are shown in the right panels in the same logarithmic greyscale. The notation is similar to that of the other figures.

eclipse map may lead to systematically underestimated fractional contribution. The reason fot this is that part of the flux from the spiral arms goes into the symmetric component because of the azimuthal smearing effect of the eclipse mapping method. The error increases for light curves of lower phase resolution: the fractional contribution of the spiral arms is better recovered when the eclipse light curve shape is better sampled. Using our simulations, we determine that the inferred fractional contribution of the spirals (40\%) may be underestimated by a factor of 1.6-2.4 for light curves $(S / N=25)$ with phase resolution of $\Delta \phi=0.010-0.015$ orbital cycles (as observed), respectively. Light curves with $S / N=50$ and $\Delta \phi=0.005$ can recover the fractional contibution of the spiral structure within $12 \%$. At small fractional contributions $(<10 \%)$, the spiral structure will start to be overestimated $(30-40 \%)$ by the technique. The error in the fractional contribution of the spirals also depends on the $S / N$ of the light curve but is much smaller than that caused by low phase resolution. Thus, since we estimated a fractional contribution of $0.15-0.3$ in Baptista et al. (2000), the true contribution of the spiral arms in those eclipse maps may be in the range $0.3-0.6$. Hence, the models with $f_{\text {spiral }} / f_{\text {total }} \sim 0.3-0.4$ should be a good approximation of the real case. In conclusion,
Table 1. Summary of simulations.

\begin{tabular}{ccccc}
\hline \hline$\left(f_{\text {spiral }} / f_{\text {total }}\right)_{\text {model }}$ & $\left(f_{\text {spiral }} / f_{\text {total }}\right)_{\text {recon }}$ & $S / N$ & $\Delta \phi$ & \\
\hline 0.40 & 0.17 & 25 & 0.015 & Fig. 3 \\
0.40 & 0.25 & 25 & 0.010 & “ \\
0.40 & 0.28 & 25 & 0.005 & “ \\
0.40 & 0.18 & 50 & 0.015 & “ \\
0.40 & 0.25 & 50 & 0.010 & “ \\
0.40 & 0.35 & 50 & 0.005 & “ \\
0.40 & 0.25 & 25 & 0.010 & Fig. 5 \\
0.30 & 0.32 & 25 & 0.010 & “ \\
0.20 & 0.13 & 25 & 0.010 & “ \\
0.10 & 0.14 & 25 & 0.010 & “ \\
0.40 & 0.25 & 50 & 0.010 & “ \\
0.30 & 0.27 & 50 & 0.010 & “ \\
0.20 & 0.19 & 50 & 0.010 & “ \\
0.10 & 0.13 & 50 & 0.010 & “
\end{tabular}

$\left(f_{\text {spiral }} / \overline{\left.f_{\text {total }}\right)_{\text {model }} \text { : is the fractional contribution in the model }}\right.$ eclipse map.

$\left(f_{\text {spiral }} / f_{\text {total }}\right)_{\text {recon }}$ : is the fraction measured by dividing the average flux in the asymmetric map by the average flux in the reconstructed eclipse map.

we can only place a lower limit to the fractional contribution of the spiral arms to the total light using eclipse maps.

\subsection{Brightness difference between the two spiral arms}

Finally, we undertake a test with a spiral structure whose arms are of unequal brightness. This is driven by the fact that observed Doppler maps show an intensity difference between the two spiral arms (Harlaftis et al. 1999; Steeghs 2001). Figure 6 presents the effect of brightness difference between the two spiral arms on the map reconstructions. We build model light curves $(\Delta \phi=0.01, S / N=50)$ from spiral structure/disk models, as described in Fig. 2 in which the red and blue arms have equal brightness (1:1, top-left panel), or the red arm is fainter - as observed - with a brightness ratio to the blue arm at 1:1.5 (middle-left panel) and finally at 1:2 (bottom-left panel). The other panels in the figure show the corresponding eclipse maps in logarithmic grayscale for three different $S / N$ ratios $(50,30$ and 20). It is notable that both arms appear in the map as a "butterfly" pattern when they have equal brightnesses regardless of the $S / N$ ratio. However, the brightness difference between the spiral arms at low $S / N$ ratio data $(<30)$ leads to loss of the fainter spiral arm in the image reconstruction.

\section{Spiral shock observations}

\subsection{Eclipse maps of the August 1994 outburst}

Morales-Rueda et al. (2000) present Doppler tomography showing persistent spiral shocks in the accretion disk of IP Peg during the August 1994 outburst. The reader is referred to Morales-Rueda et al. (2000) for the details of the observations and data reduction procedure. In summary, 164 blue spectra were obtained at a spectral resolution of $100 \mathrm{~km} \mathrm{~s}^{-1}$ at $\mathrm{H} \beta$ and a time resolution of 220 s covering the emission lines $\mathrm{H} \beta, \mathrm{H} \gamma$, 


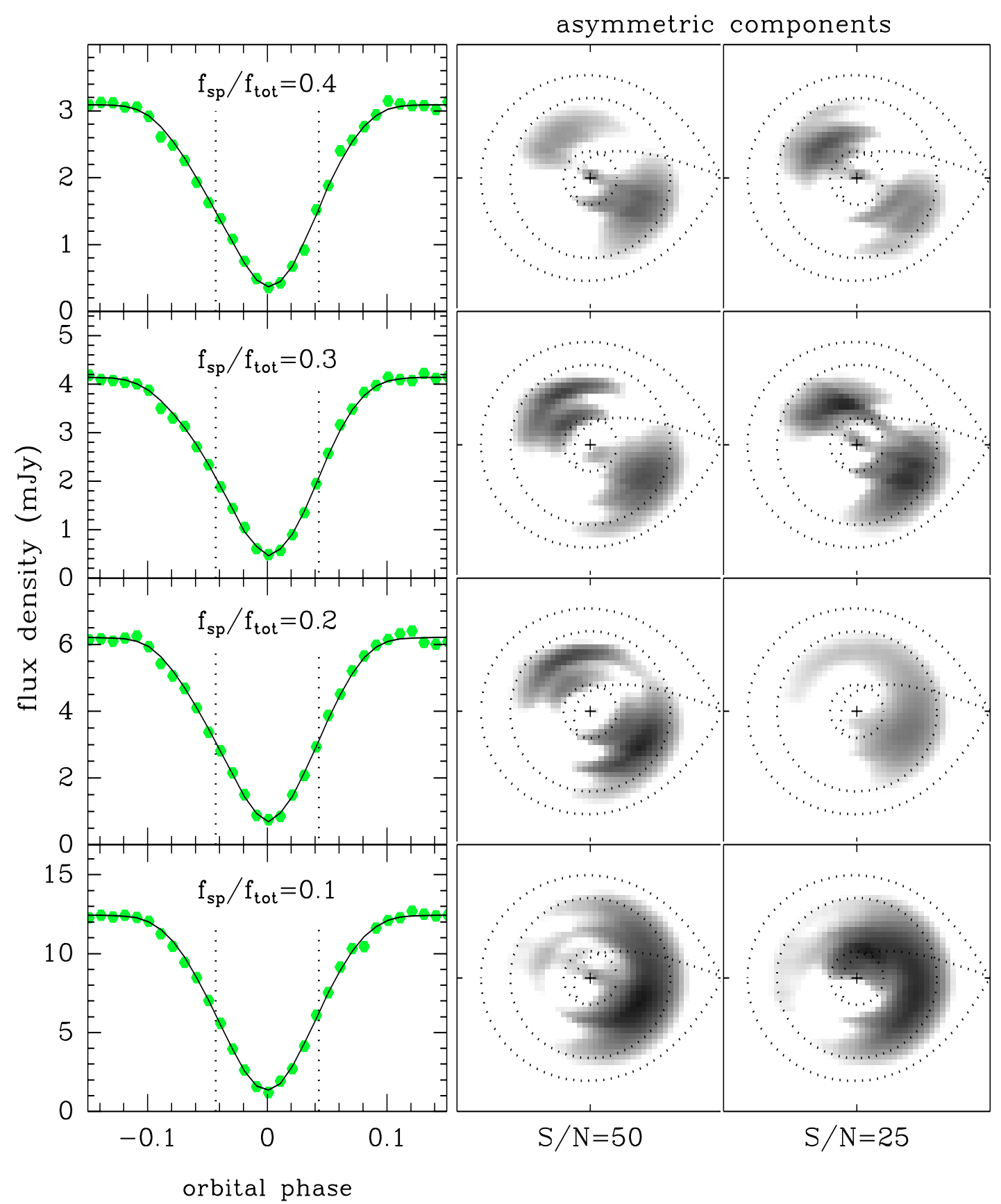

Fig. 5. The effects of dilution of the spiral structure light by a symmetric disk component on the image reconstructions. The left hand panels show model light curves $(\Delta \phi=0.01, S / N=50)$ for disk models $\left(\theta_{0}=46^{\circ}\right)$ in which the spirals contribute $40,30,20$ and 10 per cent of the total flux. The centre and right hand panels show the asymmetric parts of the corresponding eclipse maps for $S / N=50$ and 25 , respectively. When the emission from the spiral arms suffers large dilution (e.g., fractional contribution of $10 \%$ ) the method loses its ability to recover the spiral arms and returns only an asymmetric structure with a "crescent" shape at the outer disk and towards the $L_{1}$ point.

He II $4686 \AA$ (4040-4983 $\AA$ ). Here, we extend their analysis in order to include the eclipse maps for comparison with the Doppler maps. We combined the four eclipse light curves $(E=25158,25159,25164$ and 25165$)$ from the two consecutive nights the observations took place (30/31 August 1994) to produce average eclipse light curves with phase resolution of 0.010 cycles. In particular, we extracted light curves of the blue continuum and of the emission lines $\mathrm{H} \gamma$, He II $4686 \AA$, the Bowen blend and the He I 4472 A (Fig. 5 in Morales-Rueda et al. 2000) in order to reconstruct the images of the accretion disk.

We processed the light curves before applying the eclipse mapping procedure so that the continuum level before and after eclipse is the same; otherwise, artifacts are raised. These low-amplitude orbital variations were removed from the light curves by fitting a spline function to the phases outside eclipse, 


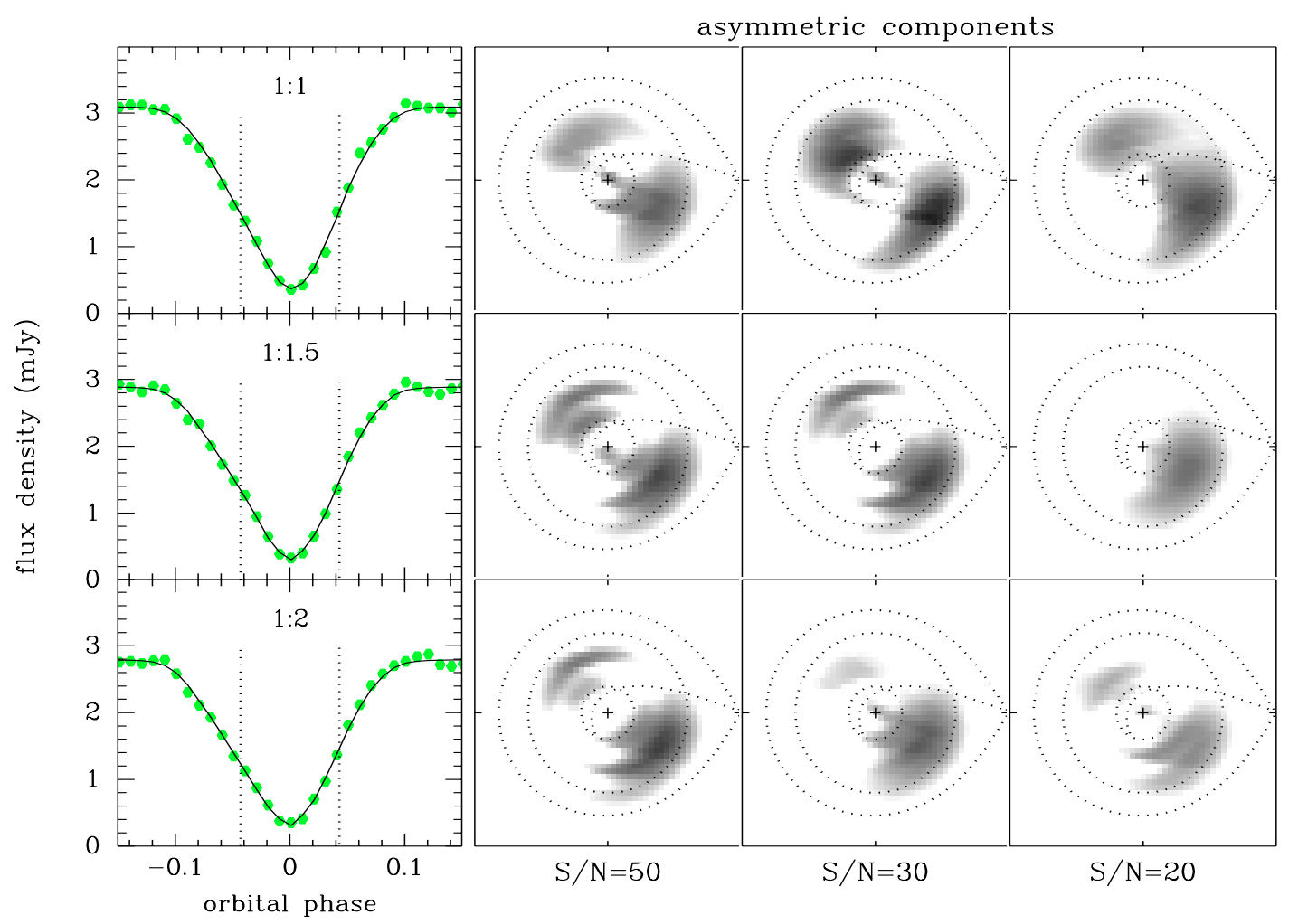

Fig. 6. The effect of brightness difference between the two spiral arms on the image reconstructions. The left-hand panels show light curves $(S / N=50)$ for models in which the red and blue arms have equal brightness (top), and models in which the red arm is fainter than the blue arm in the brightness ratio 1:1.5 (middle) and 1:2 (bottom). The right hand panels show the corresponding eclipse maps in log grayscale for three different $S / N$. Low $S / N$ data $(S / N<30)$ may lead to a loss of the fainter spiral arm. Model arms of equal brightnesses are preserved in the reconstruction map, regardless of the $S / N$.

dividing the light curve by the fitted spline, and scaling the result to the spline function at phase zero. This procedure removes orbital modulations, such as the bright spot, with only minor effects on the eclipse shape itself (e.g., Baptista et al. 2000). The other factor that affects the eclipse mapping procedure (see simulation at Fig. 4) is an accurate ephemeris for the eclipse minimum. Here, we use the linear ephemeris by Wolf et al. (1993) after correcting phase zero for mid-eclipse rather than white dwarf egress (the white dwarf eclipse width is $\Delta \phi=0.086$ cycles; Wood \& Crawford 1986)

$T_{\text {mid }}(\mathrm{HJD})=2445615.4156+0.15820616 E$

where $T_{\text {mid }}$ is the inferior conjunction of the red dwarf. IP Peg shows cyclical orbital period changes of amplitude 0.002 days (or 0.013 orbital cycles; Beekman et al. 2002). Furthemore, the scatter of eclipse timings for a given epoch is also about 0.001 days ( 0.006 cycles). Clearly, even a sinusoidal ephemeris cannot predict eclipse timings with precision better than 0.010.02 cycles for IP Peg (Wolf et al. 1993). Therefore, the best approach in order to properly phase the light curves is to center the white dwarf eclipse using contemporary quiescent eclipse timings. The August 1994 data (eclipse cycle $E \simeq 25160$ ) is luckily bracketed by two HST sets of observations of IP Peg in quiescence (Baptista et al. 1994). From these timings, we infer that the white dwarf mid-eclipse at that epoch occurred 0.008 orbital cycles before the prediction of the linear ephemeris of Wolf et al. (1993). Hence, we added +0.008 cycles to the phases of the light curves to make phase zero coincident with the inferior conjunction of the red dwarf. The minimum of the eclipse in the lines is displaced towards earlier phases in comparison to the continuum light curves. Eclipse maps were then made with three different geometries: $(i, q)=\left(81^{\circ}, 0.5\right)$ (Wood \& Crawford 1986),$(i, q)=\left(79.4^{\circ}, 0.58\right)$ (Marsh 1988) and $(i, q)=\left(86.5^{\circ}, 0.31\right)$ (Beekman et al. 2000), where $i$ is the inclination and $q$ is the mass ratio. These tests do not show any difference caused by a slight change in the adopted orbital parameters. Thus, we adopt in the following the geometry of Wood \& Crawford (1986).

All maps in Fig. 7 show an asymmetric disk. In order to resolve the asymmetric part of the disk we subtract the symmetric part of the disk and plot the residual on the right panels of the figure. The continuum eclipse is skewed towards the egress and maps into an asymmetric disk which is brighter towards the gas stream and $R_{L 1}$. The Bowen blend and $\mathrm{He}$ II high-ionization lines show a relatively narrow, asymmetric eclipse shape which maps into asymmetric brightness distributions consistent with spiral structure and similar to those in Baptista et al. (2000, see their Fig. 2). However, the Balmer and He I line maps are markedly different. The strong asymmetric Balmer emission is located close to and even outside the tidal radius, with the maximum emission at an angle of $-30^{\circ}$ with respect to the line-ofcentres. The centre of the He I eclipse occurs before that of the 


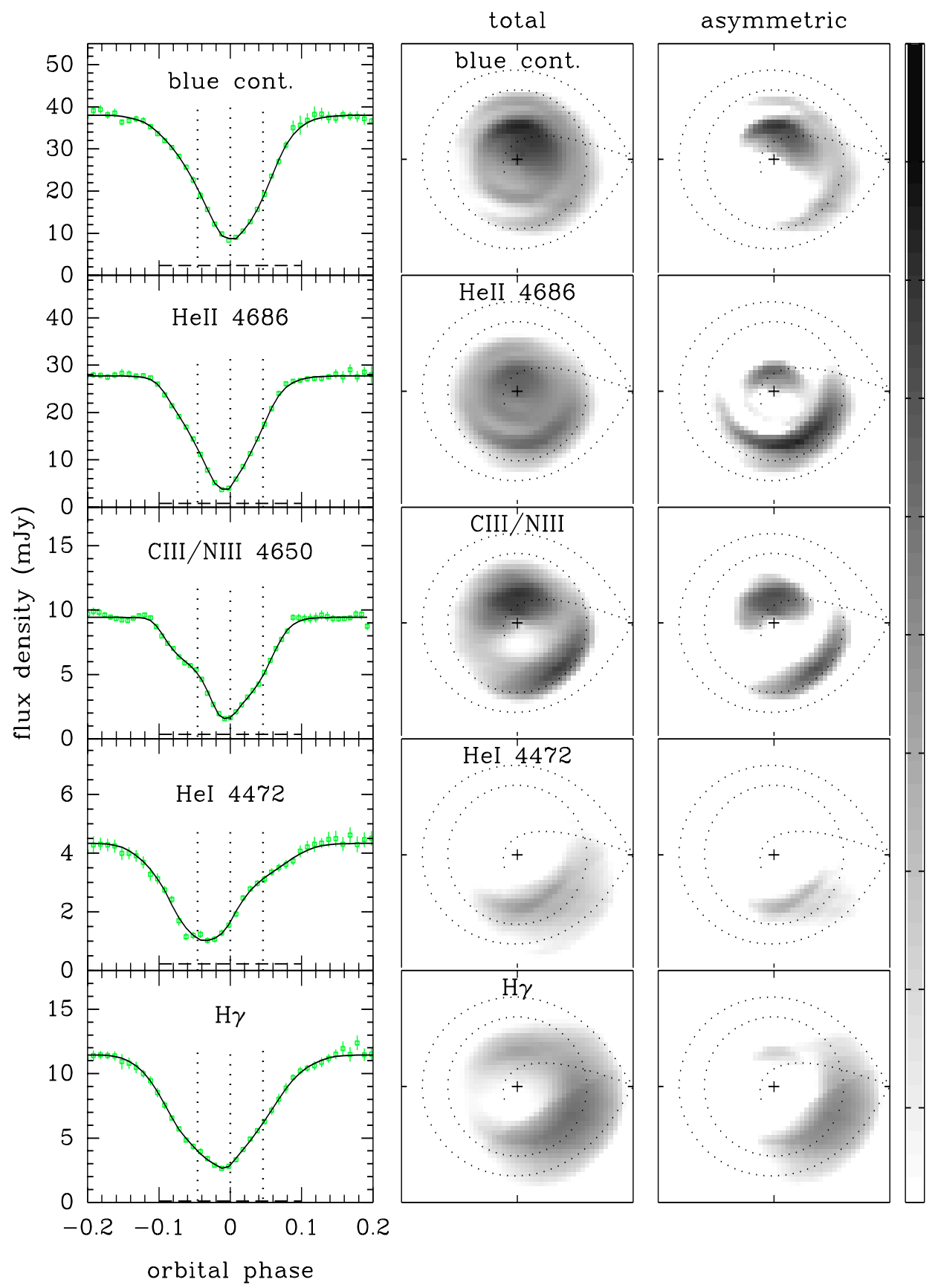

Fig. 7. The light curves, fits, eclipse maps and asymmetric parts of the maps of the blue continuum and of the emission lines He II $4686 \AA$, Bowen blend, He I $4472 \AA$ and H $\gamma$ (August 1994 outburst; Morales-Rueda et al. 2000). The middle of ingress and egress of the disk centre are marked with vertical dotted lines in the light curves and the fits to the data are also plotted. Note that the eclipse minimum varies in phase between the Balmer and helium emission lines as well as with respect to the continuum minimum. The Roche lobe of the white dwarf is plotted together with the disk radius at $0.6 R_{L_{1}}$. The donor star lies to the right of the disk map and the ballistic trajectory of the gas is also marked. The reconstruction leads to asymmetric eclipse maps in which a brighter, outer asymmetric arc is seen together with a fainter, inner arc (logarithmic grayscale). The structure resembles that of a two-spiral arm only for the Bowen and He II maps. Doppler tomography resolves clearly the two spiral arms using the out-of-eclipse data (Morales-Rueda et al. 2000).

Balmer lines, which shifts the asymmetric emission towards a direction opposite to the gas stream (blue-shifted velocities of the disk), and beyond the tidal radius. We apparently see only one of the arms in He I and there is almost no evidence of the spirals in the Balmer lines - probably because their emission is significantly diluted by other light sources in these lines (see following section on velocity-resolved light-curves). The "crescent"-shape of the brightness distribution of the Balmer lines is reminiscent of the distributions obtained for simulations where the spirals contribute only 10 per cent of the light at the corresponding wavelength (see Fig. 5). In comparison, Morales-Rueda et al. (2000) use the out-of-eclipse spectra from 
the same orbital cycles to easily resolve the spiral shocks using Doppler tomography.

\subsection{Eclipse maps from July 1998 outburst}

We also used WHT service time to obtain phase-resolved spectrophotometry with the ISIS spectrograph of an eclipse of IP Peg during its July 1998 outburst decline, four days after the peak of the outburst (18 July 1998). The 23 blue spectra (and 31 red spectra) covered the range 4407.6-4847.7 $\AA$ (and 6310.7-6717.6 $\AA$ ) at a spectral resolution of $0.2 \AA$ per pixel (and $0.8 \AA$, respectively) and with an exposure of $120 \mathrm{~s}$. The spectra covered the eclipse between 0.85 to 1.2 cycles at a phase resolution of 0.011 cycles for the red spectra and 0.014 cycles for the blue spectra. The reconstructed eclipse maps show similar distributions to the August 1994 maps, but with inferior quality which is most likely due to the poorer phase resolution and the later outburst stage (closer to quiescence which should result in a fainter spiral structure). In summary, the eclipse maps are consistent with the reconstructions from the August 1994 data (previous section) and the August 1996 data (Baptista et al. 2000).

\subsection{Velocity-resolved eclipse maps: Revealing the spiral shocks}

We investigate here velocity-resolved eclipse maps since it is a well established fact that there are non-disk emission components to the total line fluxes, for example from the donor star during outburst (Harlaftis 1999). During outburst, another contaminating line source to the disk emission is a low-velocity emission component (Harlaftis et al. 1999; Steeghs et al. 1996). Since the spiral arms and the secondary star contribute to the line profile at different Doppler velocities, it is possible to separate the contribution of these distinct light sources by suitable extraction of velocity-resolved eclipse light curves. Baptista et al. (2002) performed velocity-resolved eclipse mapping to separate the He I emission from each of the spiral arms. Here, we use this technique as a useful tool in order to separate the contribution of the low-emission components (secondary star, centre-of-binary emission; Harlaftis et al. 1999) from the disk emission and, thus, make the spirals more easily detectable in the line eclipse maps. For example, we can extract eclipse light curves from the blue (red) velocities alone. The application of the eclipse mapping technique results in the blue (red) part of the accretion disk (iso-velocity surfaces on the disk due to Keplerian rotation). The sum of the blue and red parts is indeed, as expected, consistent with the total image as built by the velocity-integrated light curve. After this test, we proceed with the aim to isolate the inner/outer disk by extracting eclipse light curves from different velocity ranges. For this purpose, we extracted Balmer and $\mathrm{He} \mathrm{I}$ light curves from the line core $(-250$ to $\left.+250 \mathrm{~km} \mathrm{~s}^{-1}\right)$ and from the line wings ( -1300 to -700 and +700 to $+1300 \mathrm{~km} \mathrm{~s}^{-1}$ ). The eclipse light curves from the core of the Balmer lines show a broad eclipse $\Delta \phi>0.2$ whose minimum is displaced towards earlier phases with respect to the continuum mid-eclipse. The eclipse light curves from the wings of the Balmer lines are centred at phase zero and show the same asymmetric eclipse shape to the He II and Bowen blend light curves with a clear step during ingress.

Figure 8 illustrates the effects of low-velocity emission components in diluting the spiral structure embedded in the accretion disk. The figure presents the results of eclipse mapping of the $\mathrm{H} \gamma$ line, from top to bottom, for the velocity-integrated total profiles (full line), the sum of the core/wings velocity regions, the core of the profiles, and the wings of the profiles. The reconstruction from the "core" light curve has a broad asymmetry, towards the $L_{1}$ point, in the outer regions of the eclipse map. The eclipse map from the "wings" light curve clearly shows the two arc-shaped spiral arms at the same orientation and azimuthal extent as those in the Bowen blend and He II eclipse maps (Fig. 7). The "core/wings" light curve (and corresponding eclipse map) is similar to the velocity-integrated light curve (and eclipse map). Similar results are found for the $\mathrm{H} \beta$ and He I lines. The phase offset from mid-eclipse and the resulting asymmetries in the eclipse maps are seen in the Balmer and He I lines (which also have a substantial contribution from the secondary star at outburst), but are not present in the He II and Bowen blend light curves and eclipse maps (the contribution of the secondary star to these lines is weak or negligible). The eclipse mapping technique, in order to account for the wide and offset eclipse, tries to build a brightness distribution in the disc regions being "eclipsed", irrespective if the physical origin lies on the secondary star or a disk wind, thereby producing a fake, broad asymmetry in the outer regions of the eclipse map displaced towards the $L_{1}$ point.

On the right-hand panels (Fig. 8), we present the $S / N$ ratio of the maps built. For each eclipse light curve, a set of 15 artificial curves was generated, in which the data points were independently and randomly varied according to a Gaussian distribution with standard deviation equal to the uncertainty at that point. The artificial curves were fitted using the eclipse mapping technique to produce a set of eclipse maps, based on random data. These, then, were combined to produce a "residuals" map by taking the pixel-to-pixel standard deviation with respect to the mean intensity. This yields the statistical uncertainty at each pixel. The uncertainty of the standard deviation for $N=15$ is $<20 \%$ which is sufficient for an illustration of the confidence limits of the eclipse map (more than an order of magnitude of Monte Carlo simulations are needed in order to reduce the uncertainty of the standard deviation down to $7 \%$ ). The result of dividing the eclipse maps of the observed data (or the mean map of the Monte Carlo simulations) by the "residuals" map is shown in the right-hand panels of Fig. 8, as maps of the inverse of the relative error or $S / N$ ratio maps. Darker regions have smaller uncertainties (greyscale is such that pixels with $S / N \leq 5$ are shown in white color, while all pixels with $S / N>100$ are shown in black color). The spiral arms in the $\mathrm{H} \gamma$ "wing" map have typical $S / N=12-20$. The $\mathrm{H} \gamma$ map (full line) has the best $S / N$ because the feeded light-curve was produced by integration over the full line profiles.

There may be various sources of low-velocity emission in the system to produce the above effects on the eclipse shape. One such emission component of unknown origin but close to the binary centre and slightly towards the gas stream has 


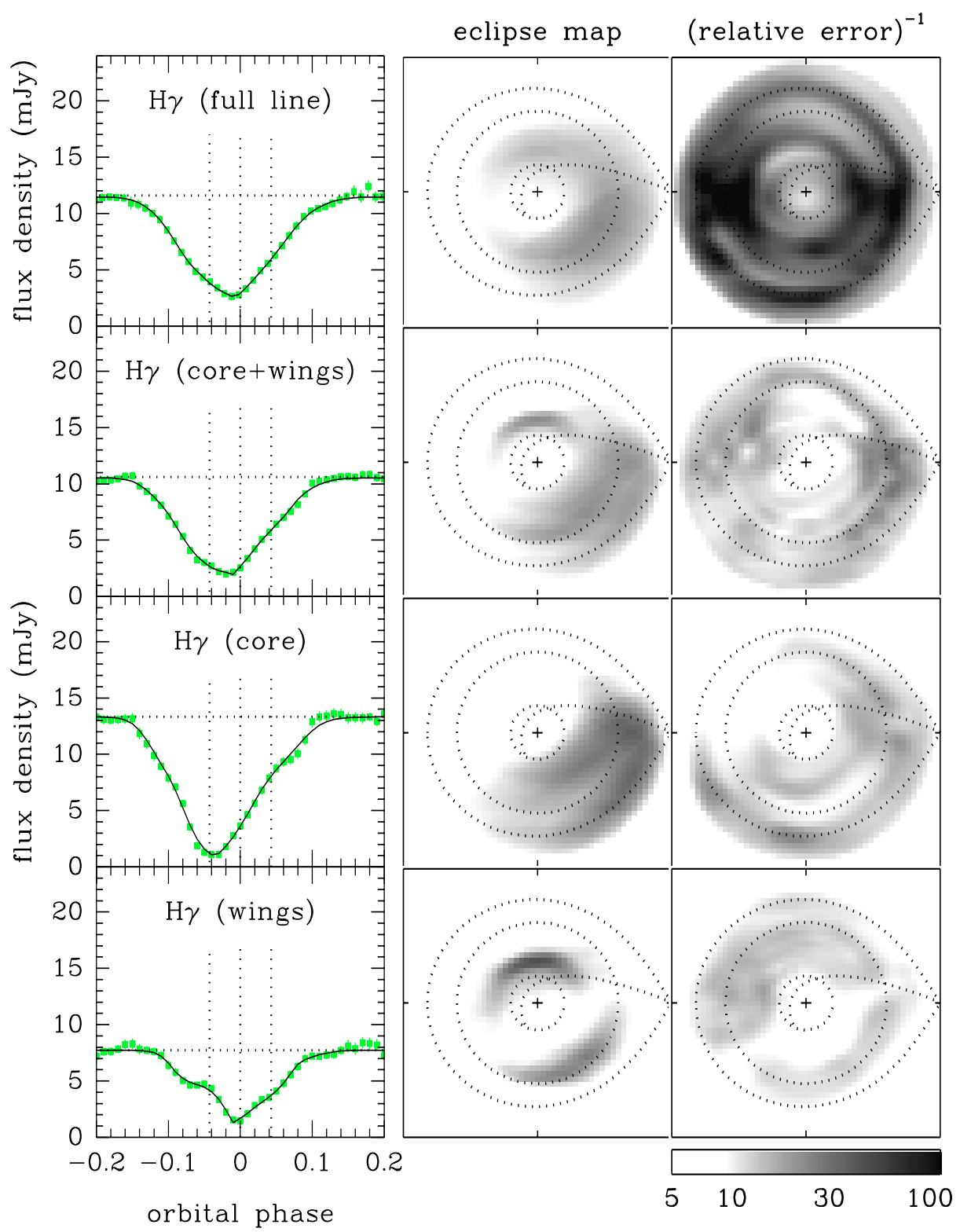

Fig. 8. Velocity-resolved (core/wings) eclipse light curves can reveal with clarity the spiral structure. The core of the H $\gamma$ line profiles $\left( \pm 250 \mathrm{~km} \mathrm{~s}^{-1}\right)$ during eclipse result in a reconstructed emission distribution towards the $L_{1}$. The wings of the $\mathrm{H} \gamma$ line profiles $( \pm 700-$ $1300 \mathrm{~km} \mathrm{~s}^{-1}$ ) during eclipse result in a reconstructed emission distribution consistent with a spiral-arm structure. The earlier mid-eclipse caused by the low-velocity emission component results in the distorted, "crescent"-like eclipse map. Lines, lobes and circles as in Fig. 7. The right-hand panels present $S / N$ ratio maps, made using Monte-Carlo simulations (pixels with $S / N \leq 5$ are painted white).

been seen at outburst maximum in IP Peg (Harlaftis et al. 1999; Steeghs et al. 1996). Wind-related emission has also been suggested by Baptista et al. (1995) in order to accommodate excess light that was not eclipsed by the companion star. The velocity of the red star, as measured from different investigators, is also low either at 280, or 300 or $330 \mathrm{~km} \mathrm{~s}^{-1}$ (Wolf et al. 1998; Marsh $\&$ Horne 1990; Beekman et al. 2000, respectively). Irradiation of the hemisphere of the star facing the white dwarf during outburst causes a significant contribution to the Balmer and He I total emission (Morales-Rueda et al. 2000), and even in He II at outburst maximum (Harlaftis et al. 1999). The implication is that if the emission pattern from the Roche lobe is not symmetric (possibly due to shielding by the bright spot and gas stream), the centre of the self-eclipse of the secondary star line emission happens earlier than the disc eclipse at phase zero. However, the data from Morales-Rueda et al. (2000) do not show any solid evidence for an asymmetric emission distribution on the Roche lobe of the companion star.

\section{Discussion}

We have performed a thorough investigation to determine the conditions under which it is possible to detect spiral structure in accretion disks of cataclysmic variables, and then compared the results with observed data. The simulations (see Table 1 for a summary) show that the phase resolution combined with the 
$S / N$ ratio are critical in resolving the features caused by the spiral shocks in the eclipse light curve shape. Indeed, poor phase resolution $(\Delta \phi>0.01)$ and low $S / N$ ratio $(<25)$ can distort the spiral structure beyond visible recognition (bottom panels in Fig. 3). In addition, the eclipse mapping technique assumes a uniform, flat accretion disk and this smears the spiral pattern into a "butterfly" pattern (top panels in Fig. 3).

Aside for the above observational parameters (time resolution and $S / N$ ) and technical effects (maximum entropy method and model assumptions), the emissivity parameters of the spiral structure are equally important in order to reveal it using the eclipse mapping technique. Dilution by the accretion disk light makes it difficult to resolve the spiral structure. The dilution of light $(>70 \%)$ can distort, through the eclipse mapping technique, the smeared spiral structure ("butterfly" pattern) to a "crescent"-like structure (bottom panels in Fig. 5). In addition to the above, the comparative emissivity between the two spiral arms may not be the same, as indeed observations indicate ("red"-arm weaker; Harlaftis et al. 1999; Steeghs 2001). This also contributes to further attenuation of the reconstructed "red" arm (bottom panels in Fig. 6). Poor phase resolution itself may result in loss of the red spiral arm even if its brightness is equal to that of the other arm. Having good phase resolution is even more important than having high $S / N$ light curves. The observed maps presented here from the early decline of the August 1994 outburst (but also similar results from spectra at late decline of the July 1998 outburst) can be more easily read given the simulations. They are consistent with reconstructing the spiral structure as a smeared and distorted spiral structure, frequently as a one-arm pattern in the accretion disk. Similarly, the maps presented by Baptista et al. (2001) at outburst maximum and by Baptista et al. (2002) at outburst decline can be interpreted in the same fashion. The WHT observations (July 1998 outburst) show an application for the case where the data does not match the requirements put forward by the simulations, and the INT observations (August 1994 outburst) is an example of a data set that meets the observational requirements.

The presence of spiral arms is inferred by small asymmetries in the shape of the eclipse light curve. When the noise in the light curve becomes comparable to the depth of these asymmetries, the method looses the ability to distinguish (or disentangle) the spiral structure from the noise. Also, the characterization of the spiral structure in the eclipse maps demands that the corresponding asymmetries in eclipse shape are properly sampled in phase. For light curves of poor phase resolution (or, more specifically, when the number of data points sampling the asymmetry caused by the spiral arm is small) it is hard to distinguish the true asymmetry from random deviations in the eclipse profile caused by noise. These effects become more important if the contribution of the spirals is diluted by other sources such as a symmetric disc component, because the relative depth (and significance) of the asymmetries decrease for increasing brightness of the additional source.

In the absence of dilution by other light sources, as with the Bowen blend light curve (Fig. 7), the spiral structures may be well recovered even for light curves of relatively poor phase resolution and incomplete phase coverage such as those of Baptista et al. (2000; see their Fig. 2). In the presence of light sources diluting the spiral structure, optimal extraction of eclipse light curves in narrow passbands matching the velocity range of the spiral arms is an interesting way to optimize the ability of the eclipse mapping method in recovering these asymmetric structures. Indeed, this is the case with the eclipse light curves of the Balmer and helium lines (Fig. 8) where an unknown source of asymmetric light shifts the mideclipse of the emission lines resulting in distorted eclipse maps ("crescent"-shape or appearance of a "single spiral-arm") with the surface brightness distribution towards the companion star. Suitable velocity-range selection from the line profiles can avoid any contamination to the spiral structure and thus resolve clearly the spiral structure in the accretion disk. Such is the case, for example, with the eclipse light curve extracted from the "wings" of the emission-line $\mathrm{H} \gamma$ profiles and the corresponding eclipse map in Fig. 8. Given the many parameters involved, it is not a surprise that the spiral structure was first discovered with the use of Doppler tomography. Doppler tomography defines very clearly the spirals since it provides more constraints from phase-resolved spectroscopy (velocity, intensity, phase).

We conclude that the eclipse mapping method is able to properly reproduce the spiral structure provided that the observations are tuned $(S / N>25$, and $\Delta \phi=0.01$ or a time resolution of at least 2 min for IP Pegasi), the data analysis is careful (avoiding any contaminating low-velocity emission by selection of suitable velocity-resolved light curves), and the physical characteristics of the spiral arms are sufficient for detection (contribution of $\geq 30 \%$ of the total disk light and minor brightness difference between the two spiral arms).

The analysis we have undertaken using eclipse maps from real and synthetic data demonstrate how future observation runs can be tailored in order to deduce in full detail the significance of the spiral structure in fast-rotating cataclysmic variable disks. The presence of spiral structure suggests that the application of eclipse mapping techniques could benefit from the use of a default model disk which smears structures along spiral patterns instead of the usual azimuthal direction (Harlaftis et al. 2004, in preparation).

Acknowledgements. The INT and WHT telescopes are operated on the island of La Palma by the Isaac Newton Group in the Spanish Observatorio del Roque de los Muchachos of the Instituto de Astrofisica de Canarias. The WHT spectra of IP Pegasi were obtained through the SERVICE programme of the Isaac Newton Group. We thank an anonymous referee for his comments. DS acknowledges a Smithsonian Astrophysical Observatory Clay Fellowship.

\section{References}

Baptista, R., Haswell, C. A., \& Thomas, G. 2002, MNRAS, 334, 198 Baptista, R. 2001, in Astrotomography: Indirect Imaging Methods in Observational Astrophysics, ed. H. Boffin, D. Steeghs, \& J. Cuypers (Heidelberg: Springer-Verlag), Lecture Notes in Phys. Ser., 573, 307

Baptista, R., Harlaftis, E. T., \& Steeghs, D. 2000, MNRAS, 314, 727

Baptista, R., Horne, K., Hilditch, R. W., Mason, K. O., \& Drew, J. E. 1995, ApJ, 448, 395 
Baptista, R., et al. 1994, ed. A. Shafter (ASP: San Francisco), ASP Conf. Ser., 56, 259

Baptista, R., \& Steiner, J. E. 1993, A\&A, 277, 331

Binney, J., \& Tremaine, S. 1988, Galactic Dynamics (Princeton University Press)

Bobinger, A., Horne, K., Mantel, K., \& Wolf, S. 1997, A\&A, 327, 1023

Hameury, J.-M. 2002, in Physics of Cataclysmic Variables and Related Objects, ed. B. T. Gaensicke, K. Beuermann, \& K. Reinsch (San Fransisco: Astronomical Society of the Pacific), ASP Conf. Ser., 261, 377

Harlaftis, E. T., Steeghs, D., Horne, K., Martín, E., \& Magazzú, A. 1999, MNRAS, 306, 348

Harlaftis, E. T. 1999, A\&A, 346, L73

Harlaftis, E. T., Marsh, T. R., Charles, P. A., \& Dhillon, V. S. 1994, 267,473

Ichikawa, S., \& Osaki, Y. 1992, PASJ, 44, 15

Ichikawa, S., \& Osaki, Y. 1994, PASJ, 46, 621

Marsh, T. R. 1988, MNRAS, 231, 1117

Marsh, T. R., \& Horne, K. 1988, MNRAS, 235, 269

Marsh, T. R., \& Horne, K. 1990, ApJ, 349, 593
Marsh, T. R., Horne, K., Schlegel, E. M., Honeycutt, R. K., \& Kaitchuck, R. H. 1990, ApJ, 364, 637

Morales-Rueda, L., Marsh, T. R., \& Billington, I. 2000, MNRAS, 313, 454

Rutten, R. G. M., van Paradijs, J., \& Tinbergen, J. 1992, A\&A, 260, 213

Steeghs, D. 2001, in Astrotomography: Indirect Imaging Methods in Observational Astrophysics, ed. H. Boffin, D. Steeghs, \& J. Cuypers (Heidelberg: Springer-Verlag), Lecture Notes in Phys. Ser., 573, 45

Steeghs, D., Harlaftis, E. T., \& Horne, K. 1997, MNRAS, 290, L28

Steeghs, D., Horne, K., Marsh, T. R., \& Donati, J. F. 1996, MNRAS, 281,626

Warner, B. 1995, Cataclysmic Variable stars (Cambridge University Press), Cambridge Astrophys. Ser., 28

Wolf, S., Barwig, H., Bobinger, A., Mantel, K.-H., \& Simic, D. 1998, A\&A, 332, 984

Wolf, S., Mantel, K. H., Horne, K., et al. 1993, A\&A, 273, 160

Wood, J. H., Marsh, T. R., Robinson, E. L., et al. 1989, MNRAS, 239, 809 